\title{
Energy Space Modeling of Power Electronics in Local Area Power Networks
}

\author{
Gregory M. Vosters and Wayne W. Weaver \\ Department of Electrical and Computer Engineering, Michigan Technological University, 121 EERC Building, \\ 1400 Townsend Dr, Houghton, MI 49931-1295, USA
}

Correspondence should be addressed to Wayne W. Weaver, wwweaver@mtu.edu

Received 17 May 2012; Revised 26 July 2012; Accepted 29 July 2012

Academic Editor: Henry S. H. Chung

Copyright ( 2012 G. M. Vosters and W. W. Weaver. This is an open access article distributed under the Creative Commons Attribution License, which permits unrestricted use, distribution, and reproduction in any medium, provided the original work is properly cited.

\begin{abstract}
Power electronics are a core enabling technology for local area power networks and microgrids for renewable energy, telecom, data centers, and many other applications. Unfortunately, the modeling, simulation, and control of power electronics in these systems are complicated when using traditional converter models in conjunction with the network nodal equations. This work proposes a change of variables for the power electronic converter models from traditional voltage and currents to input conductance and stored energy. From this change of state, a universal point of load converter model can be utilized in the network nodal equations irrespective of the topology of the converter. The only impact the original converter topology has on the new model is the bounds on the control and state variables, and the mapping back to the switching or duty cycle controls. The proposed approach greatly simplifies the modeling of local area power networks and microgrids. This simpler model can be used to study stability and energy utilization and develop high-level control strategies that were not previously feasible.
\end{abstract}

\section{Introduction}

Power electronics are increasingly being used as the interface between a local area power network, or microgrid, and the final load (FL). The wide ranges of applications include the terrestrial grid, hybrid and electric vehicles, consumer electronics, telecom systems, and many others. Interfacing point of load converters (POLCs) are used to provide controllability and act as an energy gate to an FL application. In ac power networks, the $\mathrm{dc} / \mathrm{dc}$ POLC typically also implements a power factor correction function [1] that is difficult to model as a networked systems. Even in dc power networks and microgrids, such as telecom [2], future naval electric ships [3], electric aircraft [4], computer data centers [5], and other highly sensitive and robust systems [6-8], the modeling of the power distribution network and the power electronics is not integrated. This paper proposes a modeling approach for power electronic converters that enables direct and simple model integration into the nodal equations of a power distribution network.
The POLCs are typically viewed, modeled, and controlled as a voltage translator, converting voltage levels from those provided by the source to a suitable level needed by the FL. An alternative approach is to view a POLC as an admittance translator that takes the FL impedance and reflects it to the power network. In this way, the analysis, modeling, and control synthesis of the POLCs in the local area power network becomes simpler because the admittance state of the POLC is a shunt to ground conductance in the nodal network equations. The direct application of the converter states in the network nodal equations can be used for stability analysis [9-11] or control synthesis [12, 13].

In the most general case, any power system with a switchmode converter can be simplified and viewed as the system in Figure 1 . The power system can be any combination of power sources including the terrestrial grid, batteries, combustion generators, or other power converters. The final load can be one or combinations of infinite types of loads, for example, resistive heating elements, motor drive system, or other power converters. The FL is usually modeled electrically 


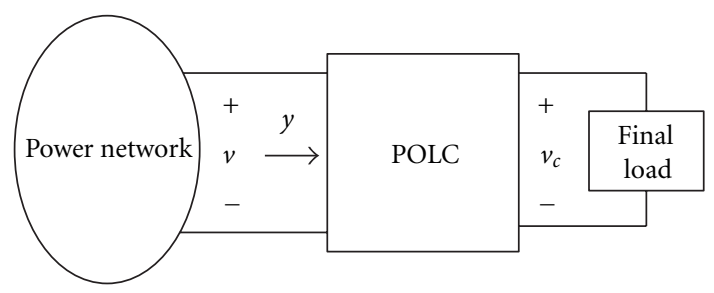

Figure 1: Point-of-load converter translates system voltage to the final load and the load impedance to the system.

as one or a combination of constant parameters, such as constant impedance or constant power. The POLC is the translator between the power system and the FL and is usually necessary to alter the form of power coming from the system to the load. This process is typically thought of as converting the voltage of the power system to a voltage suitable to the load and filtering unwanted system dynamics. However, an alternative approach is to model the POLC as translating the impedance of the load to the power system and filtering unwanted load dynamics.

A point-of-load power converter, as depicted in Figure 1, supplies power from a power network to a final load. This design and control of the process often focus on the load side of the POLC. The interaction of the POLC with the power network becomes difficult to analyze given the nature of the converter models in the voltage and current states typically used. However, a better way to approach the modeling of POLCs in a network is to utilize the power and energy flow into and out of the converter. All converters have some form of energy storage components (inductors, capacitors, and batteries), that can both absorb or supply power [14]. Then the sum total of power (neglecting losses at this point) in a POLC is illustrated in Figure 2 and described by

$$
P_{\text {in }}-P_{\text {out }}-P_{\text {store }}=0 .
$$

Power is the time rate of change in energy. Then the power stored by an energy storage component is

$$
P_{\text {store }}=\dot{w} \text {. }
$$

The power into the converter is

$$
P_{\text {in }}=v^{2} y,
$$

where $v$ is the POLC input terminal voltage and $y$ is the POLC input conductance. It is then proposed, that a power converter, of any topology, can be modeled by an energy state:

$$
\dot{w}=v^{2} y-P_{\text {out }}
$$

The input conductance of the converter $y$ depends on input voltage and current and in general has a state dependency that can be described as

$$
\dot{y}=\delta
$$

where $\delta$ is the control input to the converter model. The control variable $\delta$ can then be translated into direct switch

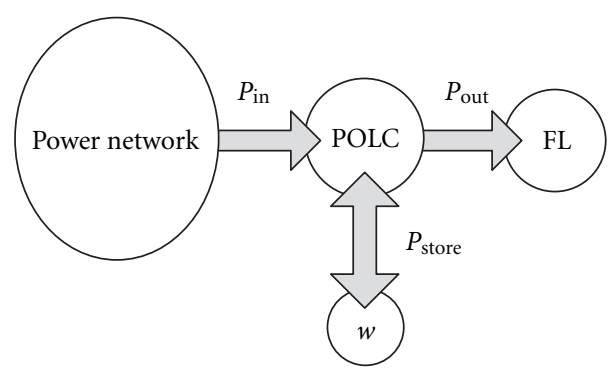

FIGURE 2: Point-of-load converter energy flow diagram with stored energy flow path.

states or average mode duty cycles of a converter, depending upon the topology being modeled.

This paper proposes the use of the two state models (4) and (5) to represent any POLC, no matter the converter topology or application of the load. This model is advantageous for use in local area power networks and microgrids since the input conductance of the POLC can be directly applied to the bus-nodal relationship:

$$
\mathbf{I}=\mathbf{Y V}
$$

of the network. Then the entire power network can be modeled as a set of differential-algebraic equations for analysis, simulation, and control synthesis.

\section{State Transformations to Energy and Conductance Space}

The energy-conductance model given in (4) and (5) can be applied to any POLC topology. However, the bounds on $\delta$ and the implementation back to the original switching control will vary by topology. In this paper, three of the most common POLC topologies will be explored, including the boost, buck, and buck-boost. As a generalized approach, the final load will be modeled as a parallel combination of constant power and constant impedance parameters.

2.1. Boost Topology. The boost topology is one of the most widely used for a network interface because of its current source input. The continuous input current lowers harmonics seen by the system and better enables power factor correction schemes in the case of ac networks [15]. The boost converter topology is shown in Figure 3 and has the switching state equations:

$$
\begin{gathered}
\dot{i}_{L}=\frac{1}{L}\left(v-(1-q) v_{c}\right), \\
\dot{v}_{c}=\frac{1}{C}\left((1-q) i_{L}-\frac{P}{v_{c}}-\frac{v_{c}}{R}\right),
\end{gathered}
$$

where the switch state $q \in\{0,1\}$. The constant power load component $P$ is modeled as a nonlinear-dependent current source. This constant power load on the switching model of the boost converter, and in fact all models presented herein, will limit the initial conditions of the model since at low 


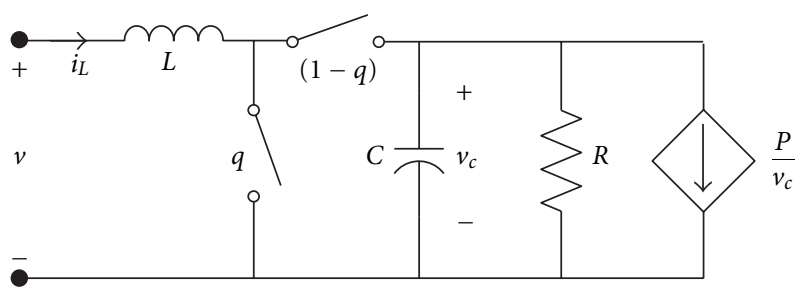

FIgURE 3: Boost converter with resistive and constant power load.

voltages this would cause excessive currents in the model. Constant power loads can present a negative incremental impedance effect on the power system, so it is desirable to use a control strategy that will utilize stored energy to promote system stability [12]. Analyzing the switching state equations, the load dynamics are only present in (8) indicating the capacitor may be used as a temporary buffer to minimize the impact on the power system. Setting the left hand side of (7) and (8) equal to zero, the average steady-state values are

$$
\begin{gathered}
\bar{v}_{c}=\frac{\bar{v}}{1-D}, \\
\bar{i}_{L}=\frac{\bar{v}}{(D-1)^{2} R}+\frac{P}{\bar{v}},
\end{gathered}
$$

where the bar-over signifies the steady-state value of a variable. As indicated in (10), the power system must eventually support the load dynamics. The input impedance can be controlled during transients if enough energy is stored within the boost converter itself. This energy can then be used to buffer the final load while presenting favorable characteristics to the network as described in [12].

2.1.1. Boost Converter Total Stored Energy State Model. To provide stability to the system, local energy storage must be used during transients while avoiding negative incremental impedance. The energy stored in a boost converter circuit is in the magnetic field of the inductor and the electric field of the capacitor. Then the total stored energy in the circuit is

$$
w_{T}=\frac{1}{2} C v_{c}^{2}+\frac{1}{2} L i_{L}^{2} .
$$

The input conductance is defined as

$$
y=\frac{i_{L}}{v} .
$$

Solving (11) and (12) for the voltage and current

$$
\begin{gathered}
v_{c}=\sqrt{\frac{2}{C}\left(w_{T}-\frac{1}{2} L(v y)^{2}\right)}, \\
i_{L}=v y .
\end{gathered}
$$

For converters in a power network, the input voltage could vary significantly given large disturbances. However for simplicity of the model derivation, the input voltage $(v)$ is assumed to be zero. Then, taking the time derivative of (13) and (14),

$$
\begin{gathered}
\dot{v}_{c}=\frac{\dot{w}_{T}-L v^{2} y \dot{y}}{\sqrt{C} \sqrt{2\left(w_{T}-(1 / 2) L(v y)^{2}\right)}}, \\
\dot{i}_{L}=v \dot{y} .
\end{gathered}
$$

Setting (15) and (16) equal to (8) and (7), respectively, then solving for the new energy and conductance state equations yield

$$
\begin{gathered}
\dot{w}_{T}=v^{2} y-\frac{2}{C R}\left(w_{T}-\frac{1}{2} L(v y)^{2}\right)-P, \\
\dot{y}=\frac{1}{L}\left(1+(q-1) \frac{1}{v} \sqrt{\frac{2}{C}\left(w_{T}-\frac{1}{2} L(v y)^{2}\right)}\right) .
\end{gathered}
$$

Solving (17) and (18) for the steady-state conditions yields

$$
\begin{gathered}
\bar{w}_{T}=\frac{C(D-1)^{2} R^{2} v^{4}+L\left((D-1)^{2} P R+v^{2}\right)^{2}}{2(D-1)^{4} R^{2} v^{2}}, \\
\bar{y}=\frac{1}{(D-1)^{2} R}+\frac{P}{\bar{v}^{2}},
\end{gathered}
$$

where $D$ is the average steady-state duty cycle of the switch $q$. By inspection, (18) shows that the input conductance does not depend upon the FL for short periods of time, allowing the POLC to use energy stored to meet transient load demand. Furthermore, the energy state is independent of switch position, $q$, so a controller could be designed based upon the conductance state equation.

2.1.2. Capacitor Energy State. Within the state equations of (17) and (18), the term

$$
w_{T}-\frac{1}{2} L(v y)^{2}
$$

is seen. The inductor current is given in (14), then (20) is the total energy minus the inductor magnetic energy and is equal to the capacitor energy. In addition, the total energy state $w_{T}$ does not appear outside the capacitor energy term. In fact, this is also the case if the total energy (inductor and capacitor) is used to model all three topologies discussed in this paper. This is because the inductor in these converter circuits primarily acts as an energy transfer component, while the true energy storage is in the electric field of the capacitor. While there is some magnetic energy storage in the inductor, it is typically smaller than the capacitive electric field energy by several orders of magnitude and thus can be neglected. Then, one way to simplify the models in Section 2.1 and (18) is to redefine the energy state as purely the capacitor energy

$$
w=w_{T}-\frac{1}{2} L(v y)^{2}=\frac{1}{2} C v_{c}^{2} .
$$

Solving for the capacitor voltage gives

$$
v_{c}=+\sqrt{\frac{2}{C} w}
$$


Then, the new energy-conduction switching model of the boost converter is

$$
\begin{gathered}
\dot{w}=v y\left((1-q) \sqrt{\frac{2}{C} w}\right)-\frac{2}{C R} w-P, \\
\dot{y}=\frac{1}{L}\left(1+(q-1) \frac{1}{v} \sqrt{\frac{2}{C} w}\right) .
\end{gathered}
$$

Setting the left-hand side of (23) equal to zero gives the steady-state conditions:

$$
\begin{aligned}
& \bar{w}=\frac{1}{2} C\left(\frac{v}{1-D}\right)^{2}, \\
& \bar{y}=\frac{1}{(1-D)^{2}} \frac{1}{R}+\frac{P}{\bar{v}^{2}} .
\end{aligned}
$$

While (23) are the switching model equations of the boost converter, the average mode model [16] can be written as

$$
\begin{gathered}
\dot{w}=v y\left((1-d) \sqrt{\frac{2}{C} w}\right)-\frac{2}{C R} w-P, \\
\dot{y}=\frac{1}{L}\left(1+(d-1) \frac{1}{v} \sqrt{\frac{2}{C} w}\right),
\end{gathered}
$$

where the average duty cycle input is a continuous variable $d \in[0,1]$. In this step, the switching dynamics and effects on the stored energy $w$ and conductance $y$ states will be lost. However, this will enable simpler modeling, simulation, and converter synthesis. The voltage and energy relationships for the boost converter are identified as

$$
(1-d) \sqrt{\frac{2}{C} w}=(1-d) v_{c}=v
$$

Then substituting (28) into (26) gives

$$
\dot{w}=v^{2} y-\frac{2}{C R} w-P,
$$

where the input power is identified in (3).

The conduction state equation (27) is a nonlinear function of the energy state $w$. However, a new control variable can be introduced as

$$
u=L \delta=1+(d-1) \frac{1}{v} \sqrt{\frac{2}{C} w}
$$

that encompasses the nonlinearity much like a feedback linearization technique described by Khalil in [17]. The conduction state equation then becomes the linear relationship

$$
\dot{y}=\frac{1}{L} u
$$

The average mode boost converter topology in the energy and conduction states is then given as (29) and (31). Note, if the input voltage variation is very small, and the load is

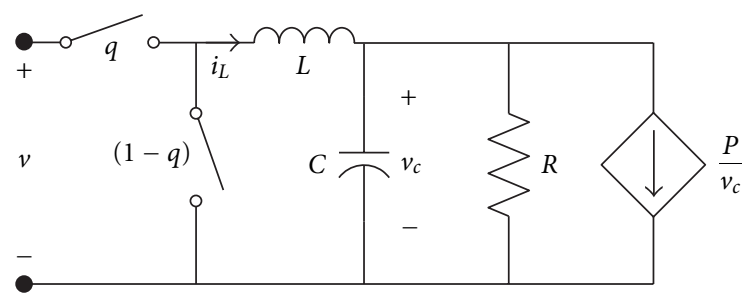

FIGURE 4: Buck converter with resistive and constant power load.

purely resistive $\left(P_{o}=0\right),(29)$ and (31) are linear with the form $\dot{x}=A x+B u$ and can be used with standard linear control techniques (pole placement, eigenvalue stability) to derive the desired control input $u$. However, the nonlinear model with variable input voltage (due to network transients) and constant power final loads $\left(P_{o}>0\right)$ and the control parameter $u$ can be synthesized from linearization or various nonlinear control techniques [17]. Nevertheless, once $u$ is determined, the duty cycle to implement the control law for a boost converter is found from

$$
d=1+(u-1) v \sqrt{\frac{C}{2 w}}=1+(u-1) \frac{v}{v_{c}} .
$$

The limits on state and control variables can be derived from the steady-state conditions (24) and (25), along with the duty cycle relationship (32) and the limits on duty cycle value. These modeling and control bounds are given in Table 1.

Another advantage of the proposed modes is that it is universal across most converter topologies. This enables the designer of a small-scale local area power network to design the system and control from the system level point of view using the energy space model without the need for explicit circuit details of the local power converters. The choice of topology and implementation of the local power converters can follow and be modified at any time after the energy space model design is complete.

2.2. Buck Topology. The buck converter topology is also a common interface to power networks to provide a step down in voltage. The general buck topology is shown in Figure 4 with the switching model state equations given as

$$
\begin{gathered}
\dot{i}_{L}=\frac{1}{L}\left(v q-v_{c}\right), \\
\dot{v}_{c}=\frac{1}{C}\left(i_{L}-\frac{P}{v_{c}}-\frac{v_{c}}{R}\right) .
\end{gathered}
$$

The energy state variable for the buck converter will be modeled as the capacitor voltage given in (21). However, since the input current to the buck converter is not continuous due to the nature of the switch $q$, it is not possible to model the input conductance as a continuous state in the switching model. Therefore, the average mode model will be applied as

$$
y=d \frac{i_{L}}{v}
$$




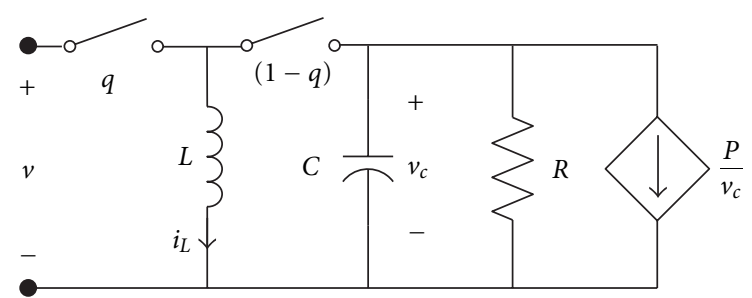

Figure 5: Buck-boost converter with resistive and constant power load.

Following the same state transformation procedures detailed in the previous section for a boost converter, the average mode model for a buck converter is found to be

$$
\begin{gathered}
\dot{w}=v^{2} y-\frac{2}{C R} w-P, \\
\dot{y}=\frac{1}{L}\left(d^{2}-d \frac{1}{v} \sqrt{\frac{2}{C} w}\right) .
\end{gathered}
$$

The steady-state conditions of (35) are

$$
\begin{gathered}
\bar{w}=\frac{1}{2} C(D v)^{2}, \\
\bar{y}=\frac{D^{2}}{R}+\frac{P}{\bar{v}^{2}} .
\end{gathered}
$$

If the control variable is defined as

$$
u=d^{2}-d \frac{1}{v} \sqrt{\frac{2}{C} w}=d^{2}-d \frac{v_{c}}{v}
$$

the transformed state model of the buck converter is the same as the boost model given in (29) and (31). The only difference is the relationship of the duty cycle and control variable $u$. The duty cycle is found from the control variable $u$ as

$$
d=\frac{v_{c}+\sqrt{4 u v^{2}+v_{c}^{2}}}{2 v} .
$$

From the defined control variable (38) and the bounds on $d$, it is seen that there is a limit on the range of the control variable $u$ and the states $w$ and $y$. In fact it can be shown that all converter topologies can be modeled by (29) and (31), with the only difference being in the bounds on the control and states. For the buck topology, the state and control bounds are found from (36), (37), and (39) and are summarized in Table 1.

2.3. Buck-Boost Topology. The buck-boost converter topology is also widely used as a power network interface in both the standard form [18] and fly-back derivative topologies [19]. The standard form buck-boost topology is shown in Figure 5 having the switching state equations:

$$
\begin{gathered}
\dot{i}_{L}=\frac{1}{L}\left(v q-(1-q) v_{c}\right), \\
\dot{v}_{c}=\frac{1}{C}\left(-(1-q) i_{L}-\frac{P}{v_{c}}-\frac{v_{c}}{R}\right) .
\end{gathered}
$$

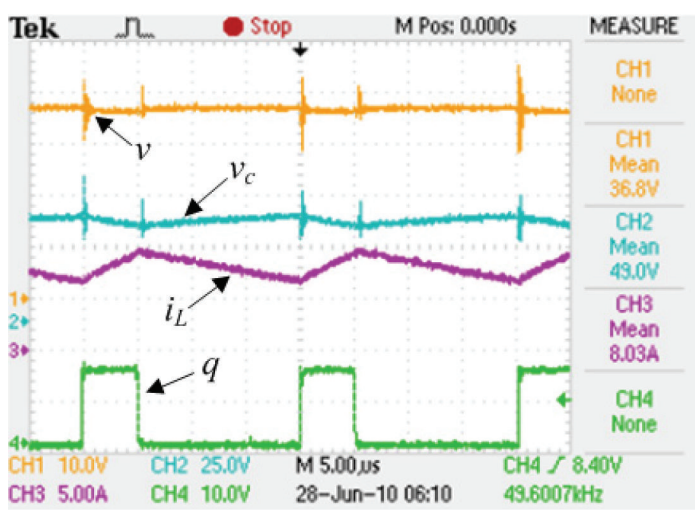

FIGURE 6: Boost converter oscilloscope switching traces for a $300 \mathrm{~W}$ load.

The energy state of the buck-boost model will again be the capacitor energy as in (21). The input current of the buck-boost converter is discontinuous, so the average mode model will be used for the input conductance as in (34). Following similar procedures from the previous sections, the transformed average mode model of the buck-boost converter becomes

$$
\begin{gathered}
\dot{w}=v^{2} y-\frac{2}{C R} w-P, \\
\dot{y}=\frac{1}{L}\left(d^{2}+\left(d-d^{2}\right) \frac{1}{v} \sqrt{\frac{2}{C} w}\right) \\
=\frac{1}{L}\left(d^{2}+\left(d-d^{2}\right) \frac{v_{c}}{v}\right)=\frac{1}{L} u
\end{gathered}
$$

which is the same form as both the buck and boost converters. The steady state condition of the energy storage and the input conductance become

$$
\begin{gathered}
\bar{w}=\frac{1}{2} C\left(\frac{D}{1-D} v\right)^{2}, \\
\bar{y}=\left(\frac{D}{1-D}\right)^{2} \frac{1}{R}+\frac{P}{\bar{v}^{2}} .
\end{gathered}
$$

The control variable in (42) is defined as

$$
u=d^{2}+\left(d-d^{2}\right) \frac{v_{c}}{v}
$$

to get the input conductance in the form proposed in (5). Then, from this definition, the resulting duty cycle of the buck-boost is

$$
d=\frac{v_{c}+\sqrt{4 u v^{2}-4 u v_{c} v+v_{c}^{2}}}{2\left(v_{c}-v\right)} .
$$

The average mode model of the buck-boost topology given in (41) and (42) are of the same form as the boost and buck converters. However the bounds on the control and state variables for the buck-boost topology are summarized in Table 1. 
TABLE 1: Control and state bounds of energy space models of three converter topologies.

\begin{tabular}{|c|c|c|c|c|c|c|}
\hline Topology & $u_{\min }$ & $u_{\max }$ & $y_{\min }$ & $y_{\max }$ & $w_{\min }$ & $w_{\max }$ \\
\hline Boost & $1-\left(v_{c} / v\right)$ & 1 & $(1 / R)+\left(P / v^{2}\right)$ & $\infty$ & $(1 / 2) C v^{2}$ & $\infty$ \\
\hline Buck & $-(1 / 4)\left(v_{c} / v\right)^{2}$ & $1-\left(v_{c} / v\right)$ & 0 & $(1 / R)+\left(P / v^{2}\right)$ & 0 & $(1 / 2) C v^{2}$ \\
\hline Buck-boost & $v_{c}^{2} / 4\left(v_{c} v-v^{2}\right)$ & 1 & 0 & $\infty$ & 0 & $\infty$ \\
\hline
\end{tabular}

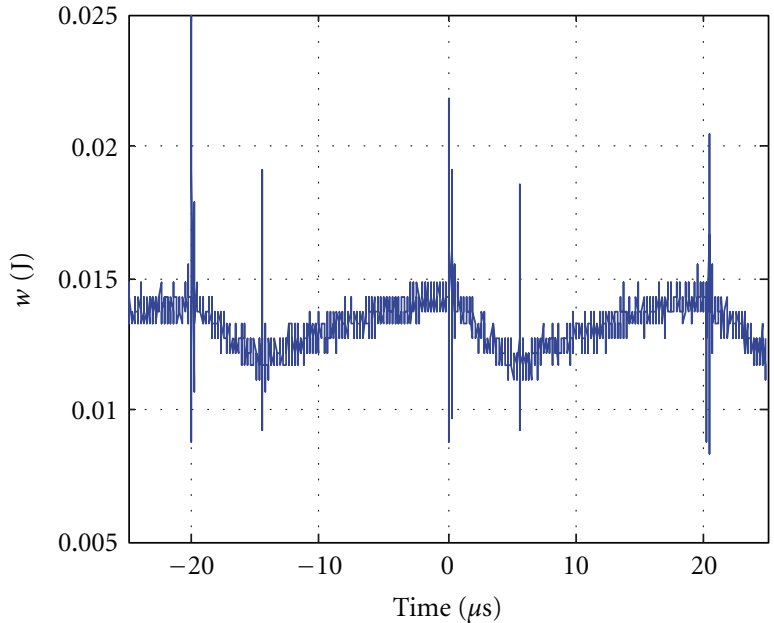

(a)

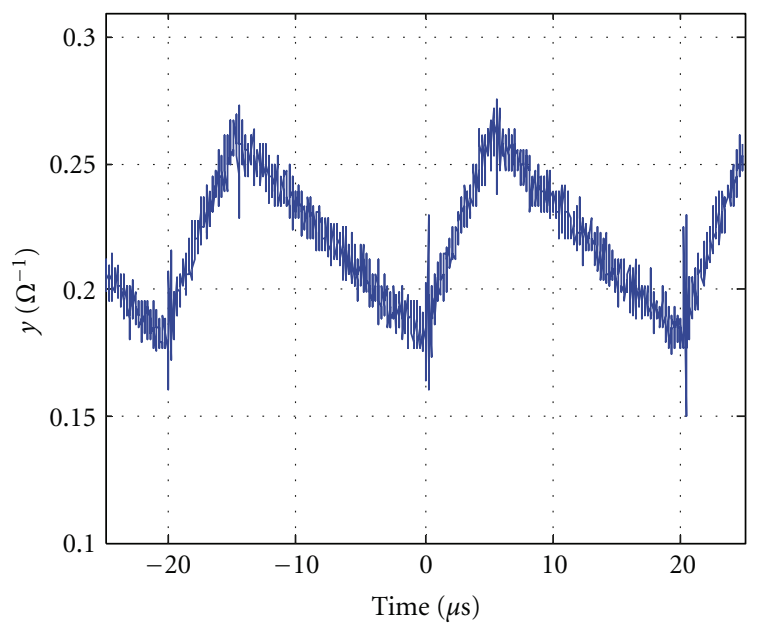

(c)

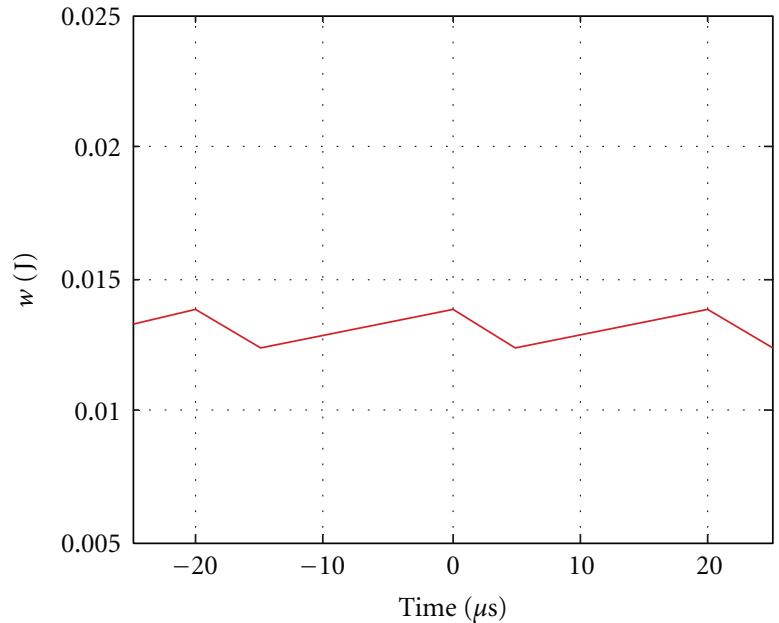

(b)

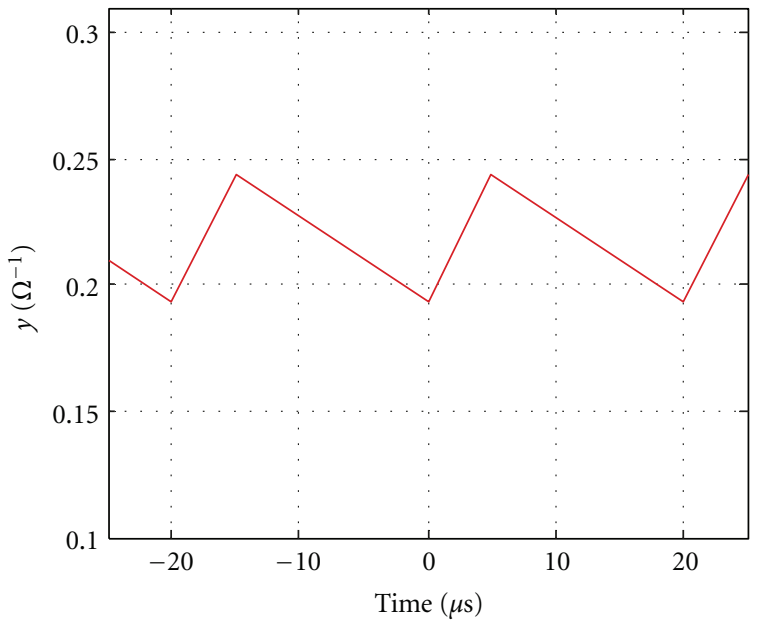

(d)

FIGURE 7: Boost converter capacitor energy and input conductance for a $300 \mathrm{~W}$ load. (a) Capacitor energy is shown for the experimental setup, (b) numeric simulation, (c) input conductance of the experimental setup, and (d) numeric simulation.

2.4. Lossy Converter Model. In the previous sections it was shown that the three converter topologies (boost, buck, and buck-boost) could be modeled with the common state equations in (29) and (31). However, this model is based on a lossless converter where, on average, the power into the converter is equal to the power out. A more accurate approach is to integrate parasitic losses, such as on-state resistances and switching losses, into the original state equations, then apply the change of variables into the proposed energy and conductance states. Nonetheless, this will lead to an energy state equation of the form

$$
w=v^{2} y-\frac{2}{C R} w-P_{\text {loss }}(w, y, t)-P
$$

despite the converter topology. However, the loss term $\left(P_{\text {loss }}(w, y, t)\right)$ will be a function of the topology and the order 


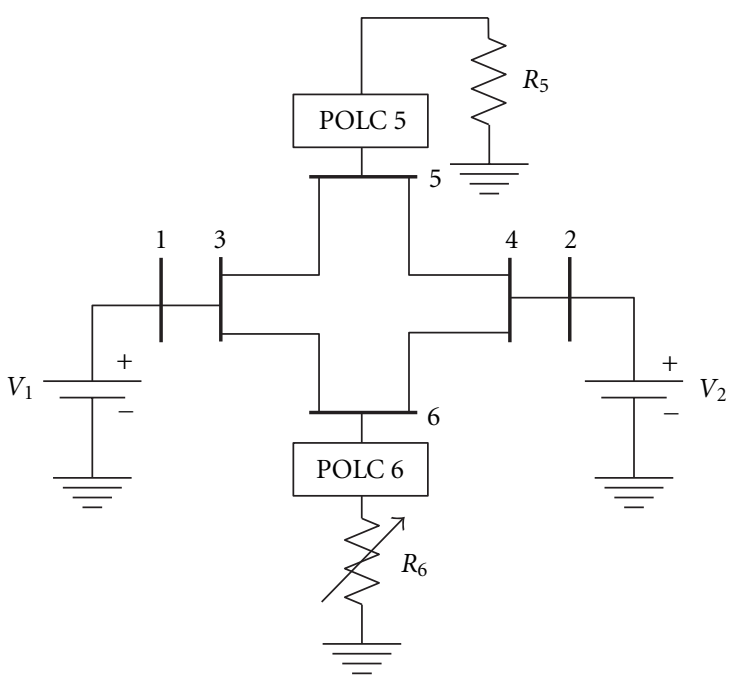

Figure 8: System model to simulate a 6-bus local area power network.

of the loss model [20]. The conductance state will also be a function of the losses, but the loss term can be included in the control variable $u$, such that the state equation is given as (31).

\section{Local Area Power Network Model}

Consider a power system with $m$ buses, $n$ sources, and a set of controllable power electronic loads $\mathcal{P}$. The bus voltages and current injections, as defined in [21], can be solved from the bus-nodal relationship (6) where $\mathbf{I}$ and $\mathbf{V}$ are the vectors of bus currents and voltages of dimension $m \times 1$. The symmetric bus admittance matrix $\mathbf{Y}$ is a function of line admittances and shunt load resistances of dimension $m \times m$. The nodal equations in (6) can be partitioned into the unknown variables of source-current injections $\left(I_{s}\right)$ and voltages of $\left(V_{s}\right)$ both of dimension $n \times 1$ and bus voltages $\left(V_{b}\right)$ of dimension $(m-n) \times 1$ :

$$
\left[\begin{array}{c}
I_{s} \\
\hline 0
\end{array}\right]=\left[\begin{array}{l|l}
Y_{11} & Y_{12} \\
\hline Y_{21} & Y_{22}
\end{array}\right]\left[\begin{array}{c}
V_{s} \\
\hline V_{b}
\end{array}\right] .
$$

The POLC voltages are $v_{i} \in V_{b}$ and the conductances are $y_{i} \in Y_{22}$, for all $i \in \mathcal{P}$. Using the standard model of a load (4) and (5) a complete set of differential-algebraic equations for a local area power network is

$$
\begin{gathered}
V_{b}=\left(Y_{22}\right)^{-1}\left(0-Y_{21} V_{s}\right), \\
I_{s}=Y_{11} V_{s}+Y_{12} V_{b}
\end{gathered}
$$

with the set of POLC equations:

$$
\begin{gathered}
\dot{w}_{i}=v_{i}^{2} y_{i}-\frac{2}{C_{i} R_{i}} w_{i}-P_{i}, \\
\dot{y}_{i}=\frac{1}{L_{i}} u_{i},
\end{gathered} \quad \forall i \in \mathcal{P},
$$

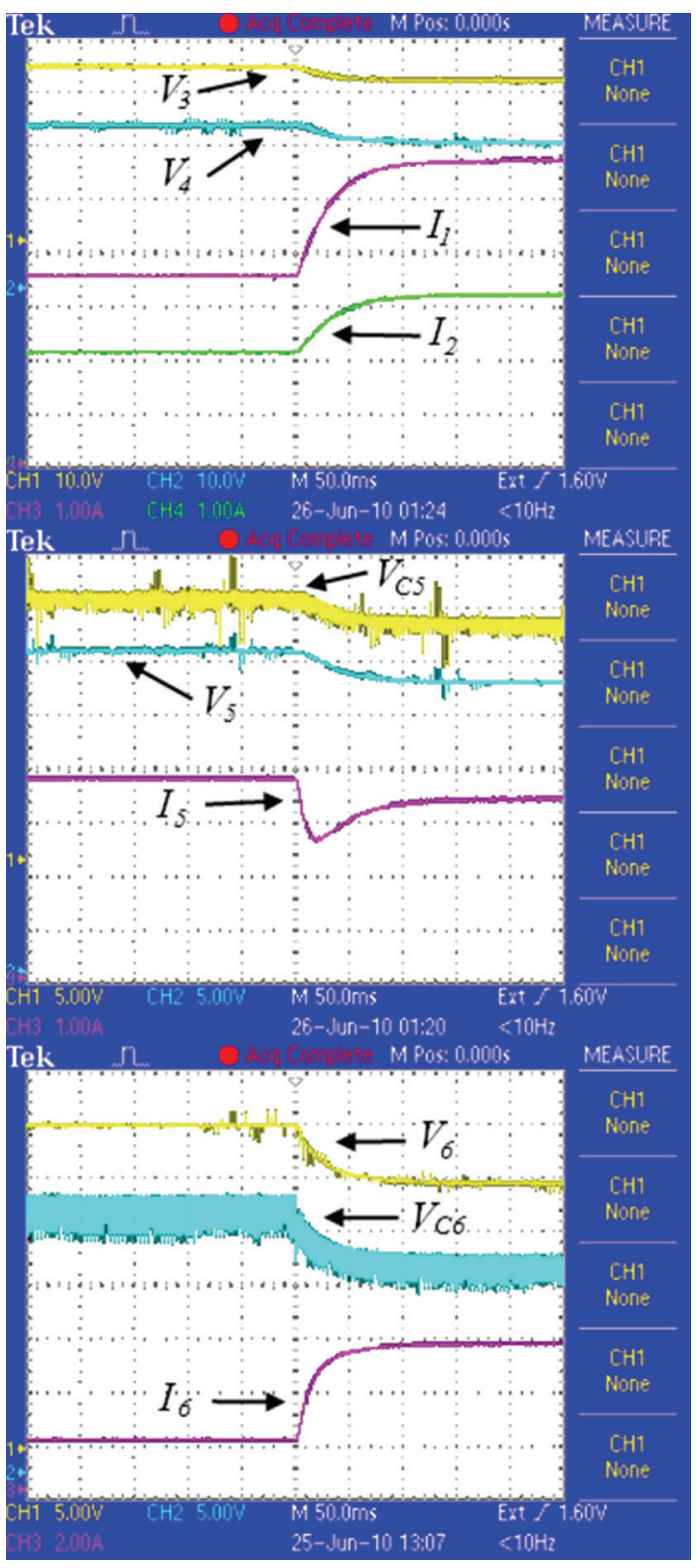

Figure 9: Oscilloscope traces from lab experiments on a local area power network. Initial conditions are $P_{5}=105 \mathrm{~W}$ and $P_{6}=50 \mathrm{~W}$, and terminal conditions are $P_{5}=90 \mathrm{~W}$ and $P_{6}=140 \mathrm{~W}$. $V_{3}$ through $V_{6}$ are the bus voltages, $I_{1}$ and $I_{2}$ are the injected source currents and, $I_{5}$ and $I_{6}$ are the load currents from the system in Figure 8.

The nodal network equations (48) and generalized POLC model (49) form a set of differential-algebraic equations that can be easily simulated and used for control synthesis, system stability studies, and planning. In this paper, only the POLCs are transformed into the energy and input conductance models, while the sources are modeled as constant voltages. However, this proposed approach can also be extended to model both power electronics POLC and energy sources with power electronic network interfaces, such as renewable energy systems. 


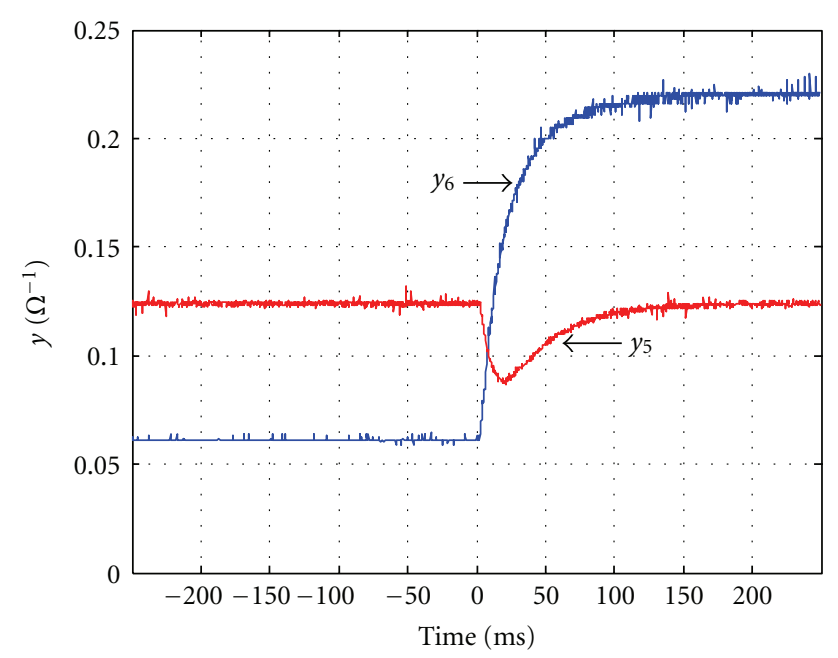

Figure 10: Input conductances of POLC5 and POLC6 during 6-bus laboratory experiment.

TABLE 2: Boost converter experimental values for $300 \mathrm{~W}$ resistive load.

\begin{tabular}{lcccc}
\hline $\bar{v}$ & $\bar{v}_{c}$ & $D$ & $C$ & $R$ \\
\hline $36 \mathrm{~V}$ & $48 \mathrm{~V}$ & 0.25 & $11 \mu \mathrm{F}$ & $8.1 \Omega$ \\
\hline
\end{tabular}

\section{Illustrative Examples}

Two lab experiments have been conducted to demonstrate the models developed within this paper. First, a simulation and experimental results for a single, boost converter demonstrate the switching model. Then a small bench-scale local area power network will demonstrate the proposed model integration. Numeric simulations will estimate the bus voltages and currents for a 6-bus system. The steady state model of the buck converter will be used to estimate the conductance at two points of load. After completing a numeric simulation, the results are compared to experimental data to verify both the steady state buck converter model and the local area power network model.

4.1. Boost Converter. The switching state equations for the boost converter are the basis for the average mode model and the steady state model. This indicates the switching equations should be validated before attempting to verify the steady state model. A numeric simulation was created using the switching equations described in (17) and (18). The numeric simulation does not include converter losses and models only the differential-algebraic equations for the input conductance and the capacitor energy states. To form a good comparison, both the simulation and the experimental setup are shown in steady state. This will show the switching dynamics when viewed at a small enough time scale, while also providing verification of the steady state equations (24) and (25).
A boost converter was constructed as shown in Figure 3 with the parameters given in Table 2 for a constant resistance load of $300 \mathrm{~W}$. The control of the hardware was implemented with a PWM process operating at $50 \mathrm{kHz}$ and a PI feedback loop around the output capacitor voltage $v_{c}$. The oscilloscope image for this experiment in Figure 6 with the data processed into conductance and energy is shown alongside with the numeric simulation shown in Figure 7. The graphs indicate both the conductance and the energy storage have larger peak values than the simulated converter. However, the results are within a reasonable error and indicate the energyconductance switching model is valid. The error can be accounted for in converter losses since more energy will be needed to overcome losses, resulting higher peak to peak values. From these images, the steady state conditions are calculated. The theoretical steady state capacitor energy is calculated from (24) with the values in Figure 7 to be $0.0132 \mathrm{~J}$. From the experimental data, the average capacitor voltage is $49.0 \mathrm{~V}$. The deviates from the nominal of $48 \mathrm{~V}$ are due to two possible reasons, a miscalibration of the PI feedback control, or a measurement error of the oscilloscope shown in Figure 6. The high-frequency spikes in the voltage waveforms of Figure 6 are due to the commutation of the mosfet switch. For a value of $49 \mathrm{~V}$, the average energy stored is $0.0132 \mathrm{~J}$ from (21). The theoretical steady state input conductance is calculated to be $0.2195 \Omega^{-1}$. The experimental values of conductance, shown in Figure 7 , are used in (25), whereas the experimental result is $0.2185 \Omega^{-1}$ from the average input voltage and inductor current. These results indicate the steady state model is also correct since the error is almost negligible.

4.2. Local Area Power Network Model. The model developed for the local area power network is useful for localized applications of power converters, such as a ship, a hybrid electric vehicle, or a telecom network. Transient load demand at one point of the network can cause changes throughout the network in terms of bus voltages and currents. Due to the complexity of this type of system, it is desired to quantify effects of transient load demand analytically. Therefore, a 6bus local area power network was created to analyze and demonstrate (48) and (49), providing a reasonable level of complexity to the system while also not becoming too cumbersome to simulate numerically.

The 6-bus power network shown in Figure 8 was built in the laboratory with line conductances given in Table $3 . V_{1}$ and $V_{2}$ maintain bus 1 and 2 voltages at a constant $36 \mathrm{~V}_{\mathrm{dc}}$. $V_{3}$ through $V_{6}$ are the bus voltages; $I_{1}$ and $I_{2}$ are the injected source currents at bus 1 and 2 .

The POLCs at both bus 5 and $6(\mathcal{P}=\{5,6\})$ are constant duty cycle buck converters with $D=0.8$. The steady state model of the power network generates the bus admittance matrix (47) and can be solved for using a numeric solver. The numeric results will be compared to experimental lab results.

To simulate the system using the bus admittance matrix, the steady state condition will be analyzed. The system in Figure 8 is allowed to reach steady state conditions, then the 
TABLe 3: Bus conductances for the 6-bus local area power network.

\begin{tabular}{lcccccc}
\hline Line & $Y_{13}$ & $Y_{24}$ & $Y_{35}$ & $Y_{36}$ & $Y_{45}$ & $Y_{46}$ \\
\hline$Y$ & $0.8547 \Omega^{-1}$ & $0.3438 \Omega^{-1}$ & $0.6897 \Omega^{-1}$ & $0.6061 \Omega^{-1}$ & $1.938 \Omega^{-1}$ & $0.8547 \Omega^{-1}$ \\
\hline
\end{tabular}

TABLE 4: Local area power network model numeric solutions and measured values. In case (A), $R_{6}$ is $11.2 \Omega$ with a load power $P_{6}=50 \mathrm{~W}$. In case (B) $R_{6}$ is $2.8 \Omega$ with a load power $P_{6}=140 \mathrm{~W}$.

\begin{tabular}{|c|c|c|c|c|c|c|c|}
\hline & & $I_{1}$ & $I_{2}$ & $V_{3}$ & $V_{4}$ & $V_{5}$ & $V_{6}$ \\
\hline \multirow{2}{*}{ (A) } & Numeric result & $3.43 \mathrm{~A}$ & $1.76 \mathrm{~A}$ & $31.98 \mathrm{~V}$ & $30.94 \mathrm{~V}$ & $30.36 \mathrm{~V}$ & $30.19 \mathrm{~V}$ \\
\hline & Lab result & $3.48 \mathrm{~A}$ & $2.04 \mathrm{~A}$ & $32.4 \mathrm{~V}$ & $30.4 \mathrm{~V}$ & $29.8 \mathrm{~V}$ & $30.0 \mathrm{~V}$ \\
\hline \multirow{2}{*}{ (B) } & Numeric result & $5.75 \mathrm{~A}$ & $2.96 \mathrm{~A}$ & $29.28 \mathrm{~V}$ & $27.51 \mathrm{~V}$ & $27.34 \mathrm{~V}$ & $24.42 \mathrm{~V}$ \\
\hline & Lab result & $5.60 \mathrm{~A}$ & $3.08 \mathrm{~A}$ & $30.0 \mathrm{~V}$ & $27.2 \mathrm{~V}$ & $27.0 \mathrm{~V}$ & $25.0 \mathrm{~V}$ \\
\hline
\end{tabular}

end-resistive load of POLC6 is increased at $t=0$, described by

$$
\begin{gathered}
R_{5}=5.6 \Omega, \\
R_{6}= \begin{cases}11.2 \Omega & t<0, \\
2.8 \Omega & t \geq 0 .\end{cases}
\end{gathered}
$$

This will simulate a step increase in load power at POLC6 and will show how load dynamics can influence the power system and the dynamic response of the converters and the proposed load model. The average mode input conductances of POLC5 and POLC6, respectively, are calculated from (37) as

$$
\begin{gathered}
Y_{5}=0.1143 \Omega^{-1}, \\
Y_{6}= \begin{cases}0.0571 \Omega^{-1} & t<0, \\
0.2286 \Omega^{-1} & t \geq 0 .\end{cases}
\end{gathered}
$$

The numeric solution to this problem is solved using the conductance obtained in (51) along with the conductances in Table 3 to generate the bus admittance matrix. By numerically solving the system as described in (48) and (49), the bus voltages and currents are calculated. The experimental results in Table 4 indicate the steady state conductance of the buck converter in (37) is an accurate model since the bus voltages and currents nearly match the experimental results. Small errors exist and are believed to be related to converter losses not modeled in the numeric solution and experimental error.

The oscilloscope traces from the lab experiment are shown in Figure 9. Three separate oscilloscopes were used to capture the data, using a common trigger. The initial loads are $P_{5}=105 \mathrm{~W}$ and $P_{6}=50 \mathrm{~W}$ and switch to $P_{5}=90 \mathrm{~W}$ and $P_{6}=140 \mathrm{~W}$ at $t>0 \mathrm{~s}$. Due to large input capacitance on the buck converters used for this experiment, bus voltages $V_{5}$ and $V_{6}$ do not drop rapidly once the load power is increased. Future experiments should be completed with lower input capacitance on the POLC. In this experiment, the power supplies at buses 1 and 2 regulate the voltages $V_{1}$ and $V_{2}$, respectively. However all other bus voltages are unregulated and vary with changes in network current.

The change in conductance for this experiment is demonstrated in Figure 10 using the data captured from the oscilloscopes in Figure 9. When the experimental conductances in Figure 10 are compared to the theoretical values calculated in (51), it shows the steady state models accurately predict the system behavior. The POLC input conductance $Y_{5}$ returns to its initial state at $0.1229 \Omega^{-1}$, while the conductance $Y_{6}$ increases from $0.0613 \Omega^{-1}$ to $0.2193 \Omega^{-1}$. These values closely match the expected values and are well within experimental error.

\section{Conclusions}

Modeling, simulation, and control synthesis are complicated using traditional models of power electronics and network equations. This paper has presented a method that uses a change of state variables of a power electronic converter from traditional voltage and currents to conductance and energy. From this change of state, a universal point of load converter model can be used in the network nodal equations irrespective of the topology of the original converter model. The only impact the original converter topology has on the new model is the bounds on the control and state variables and the translation back to the switching or duty cycle signals. The proposed approach greatly simplifies the modeling of local area power networks and microgrids. This simpler model can be used to study stability and energy utilization and develop high-level control strategies that were not previously feasible.

\section{Acknowledgment}

This paper is based upon work supported by the National Science Foundation under Grant no. 0901094.

\section{References}

[1] I. Yamamoto, K. Matsui, and M. Matsuo, "A comparison of various DC-DC converters and their application to power factor correction," in Proceedings of the Osaka Power Conversion Conference, pp. 128-135, 2002.

[2] A. Kwasinski and P. T. Krein, "Optimal configuration analysis of a microgrid-based telecom power system," in Proceedings of the 28th Annual International Telecommunications Energy Conference (INTELEC '06), pp. 1-8, September 2006. 
[3] J. G. Ciezki and R. W. Ashton, "Selection and stability issues associated with a navy shipboard DC zonal electric distribution system," IEEE Transactions on Power Delivery, vol. 15, no. 2, pp. 665-669, 2000.

[4] L. Han, J. Wang, and D. Howe, "Stability assessment of distributed Dc power systems for "more-electric" aircraft," in Proceedings of the 4th IET International Conference on Power Electronics, Machines and Drives (PEMD '08), pp. 661-665, York, UK, April 2008.

[5] A. Pratt, P. Kumar, and T. V. Aldridge, "Evaluation of $400 \mathrm{~V}$ DC distribution in telco and data centers to improve energy efficiency," in Proceedings of the International Telecommunication Energy Conference (INTELEC '07), pp. 32-39, Rome, Italy, October 2007.

[6] D. Salomonsson and A. Sannino, "Low-voltage DC distribution system for commercial power systems with sensitive electronic loads," IEEE Transactions on Power Delivery, vol. 22, no. 3, pp. 1620-1627, 2007.

[7] H. Kakigano, Y. Miura, T. Ise, and R. Uchida, "DC voltage control of the dc micro-grid for super high quality distribution," in Proceedings of the 4th Power Conversion ConferenceNAGOYA (PCC-NAGOYA '07), pp. 518-525, Nagoya, Japan, April 2007.

[8] F. Bodi, “'DC-grade' reliability for UPS in telecommunications data centers," in Proceedings of the International Telecommunication Energy Conference (INTELEC '07), pp. 595-602, Rome, Italy, October 2007.

[9] S. D. Sudhoff and S. F. Glover, "Admittance space stability analysis of power electronic systems," IEEE Transactions on Aerospace and Electronic Systems, vol. 36, no. 3, pp. 965-973, 2000.

[10] L. Harnefors, M. Bongiorno, and S. Lundberg, "Stability analysis of converter-grid interaction using the converter input admittance," in Proceedings of the European Conference on Power Electronics and Applications (EPE '07), pp. 1-10, Aalborg, Denmark, September 2007.

[11] M. Belkhayat, R. Cooley, and A. Witulski, "Large signal stability criteria for distributed systems with constant power loads," in Proceedings of the 26th Annual IEEE Power Electronics Specialists Conference, pp. 1333-1338, June 1995.

[12] W. W. Weaver and P. T. Krein, "Optimal geometric control of power buffers," IEEE Transactions on Power Electronics, vol. 24, no. 5, pp. 1248-1258, 2009.

[13] P. Gupta and A. Patra, "A stable energy-based control strategy for DC-DC boost converter circuits," in Proceedings of the IEEE 35th Annual Power Electronics Specialists Conference (PESC '04), vol. 5, pp. 3642-3646, June 2004.

[14] R. S. Balog, W. W. Weaver, and P. T. Krein, "The load as an energy asset in a distributed architecture," in Proceedings of the 1st IEEE Electric Ship Technologies Symposium, pp. 261-267, July 2005.

[15] O. García, J. A. Cobos, R. Prieto, P. Alou, and J. Uceda, "Single phase power factor correction: a survey," IEEE Transactions on Power Electronics, vol. 18, no. 3, pp. 749-755, 2003.

[16] P. T. Krein, J. Bentsman, R. M. Bass, and B. L. Lesieutre, "On the use of averaging for the analysis of power electronic systems," IEEE Transactions on Power Electronics, vol. 5, no. 2, pp. 182-190, 1989.

[17] H. K. Khalil, Nonlinear Systems, Prentice Hall, Upper Saddle River, NJ, USA, 3rd edition, 2002.

[18] G. K. Andersen and F. Blaabjerg, "Current programmed control of a single-phase two-switch buck-boost power factor correction circuit," IEEE Transactions on Industrial Electronics, vol. 53, no. 1, pp. 263-271, 2006.
[19] J. M. Alonso, M. A. Dalla Costa, and C. Ordiz, "Integrated buck-flyback converter as a high-power-factor off-line power supply," IEEE Transactions on Industrial Electronics, vol. 55, no. 3, pp. 1090-1100, 2008.

[20] A. Davoudi, J. Jatskevich, and P. L. Chapman, "Computeraided dynamic characterization of fourth-order PWM DC-DC converters," IEEE Transactions on Circuits and Systems II, vol. 55, no. 10, pp. 1021-1025, 2008.

[21] A. R. Bergen and V. Vittal, Power Systems Analysis, Prentice Hall, Upper Saddle River, NJ, USA, 2nd edition, 2000. 

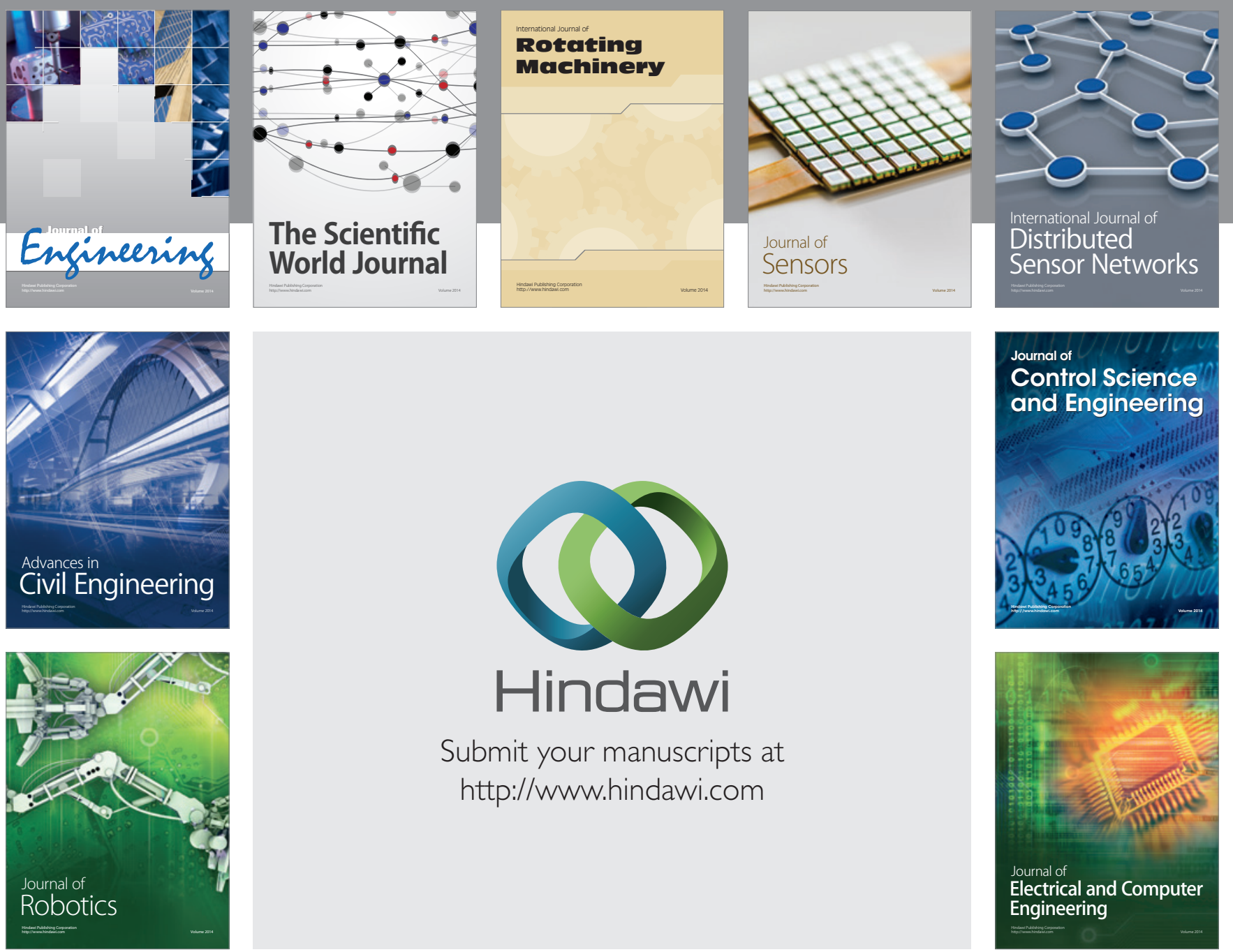

Submit your manuscripts at

http://www.hindawi.com
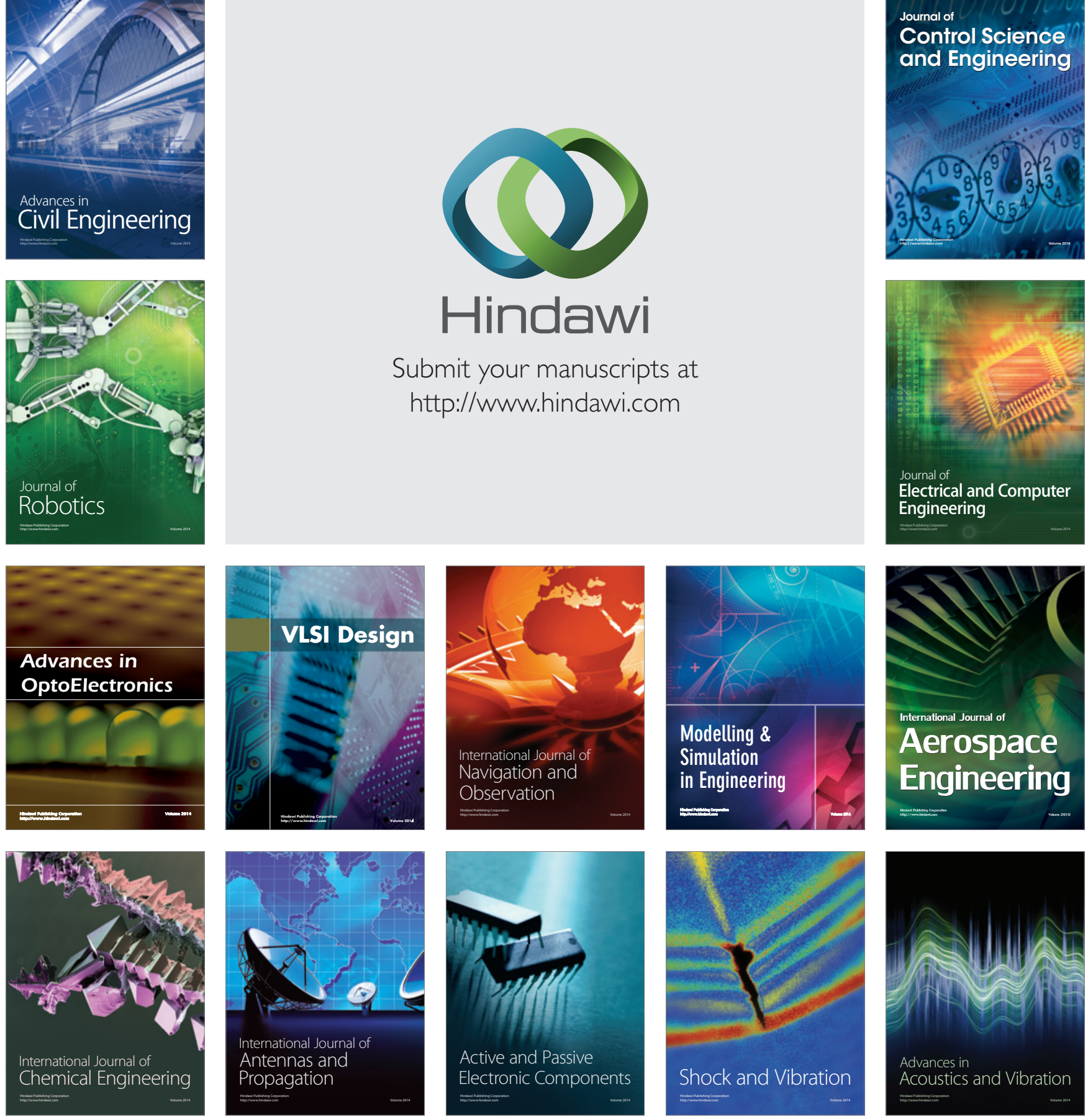